\title{
Effects of Preceding Birth Intervals on child mortality in Ethiopia; Evidence from the Demographic and Health Surveys, 2016
}

\section{Tariku L*}

School of public health, Wolaita Sodo University, Ethiopia

*Corresponding author: Tariku Laelago, School of public health, Wolaita Sodo University, Wolaita Sodo, Ethiopia, Tel: +251913400247; Email: tarikulalago@gmail.com

\section{Research Article}

Volume 3 Issue 1

Received Date: March 01, 2019

Published Date: March 12, 2019

DOI: $10.23880 /$ eij-16000118

\section{Abstract}

Background: Reducing deaths of newborn and children under 5 years of age became an agenda. The effect of preceding birth interval on child mortality was evidenced by different findings. The objective of this study was assessing the effects of preceding birth interval on child mortality in Ethiopia.

Methods: The study participants were 8460 non-first births in the 5 years before the 2016 EDHS (Ethiopian demographic health survey). Data analysis was done by using SPSS version 20. Bivariate and multivariate logistic regression analysis was done to assess the relationship between dependent and independent variables. Odds ratio at $95 \% \mathrm{CI}$ was computed to assess the strength of association between dependent and independent variables. Variables with P-value $<0.05$ in multivariate analysis was used to declare significance of association.

Result: Child whose proceeding birth interval was less than 18 months (AOR= 2.769; CI 95 \%( 1.956, 3.921)) and child whose preceding birth interval was between 18 and 23 months (AOR=1.500; 95 \% CI $(1.038,2.168)$ ) were more likely to die than child with birth interval of 60 months and above. Child whose preceding birth interval was between 36 and 47 months $(\mathrm{AOR}=0.663,95 \% \mathrm{CI}(.446, .986))$ was less likely to die than child with birth interval of 60 months and above.

Conclusion: The preceding birth interval and child mortality was significantly associated. Child whose preceding birth interval less than 18 months, and 18 to 23 months were more likely to die than child with birth interval of 60 months and above. Child whose preceding birth interval was between 36 and 47 months was less likely than to die than child with birth interval of 60 months. Health education on effect of short preceding birth interval should be given by health workers. Promotion of family planning should be strengthened at all levels.

Keywords: Interval; Preceding Birth; Child Mortality

Abbreviations: DHS: Demographic and Health Survey; EDHS: Ethiopian Demographic Health Survey;
EA: Enumeration Area; HH: Household. 


\section{Epidemiology international journal}

\section{Background}

The world made incredible progress in child survival in the past few decades. Millions of children have better survival chances than in 1990, where 1 in 26 children died before reaching age 5 in 2017, compared to 1 in 11 in 1990. Additionally, progress in reducing child mortality has been hastened in the 2000-2017 compared with the 1990s, with the annual rate of reduction in the global under-five mortality rate increasing from 1.9 percent in 1990-2000 to 4 per cent in 2000-2017. In spite of the global progress in reducing child mortality over the past few decades, an estimated 5.4 million children under age 5 died in 2017-roughly half of those deaths happened in sub-Saharan Africa [1].

In Ethiopia, under-five mortality rate per 1000 live births decreased from 205 in 1990 to 67 in 2015. Over the same time interval, the infant mortality rate declined from 123 to 48 and the neonatal mortality rate also showed a similar decline from 54 to 20.The target for under-five mortality rate was accomplished, while the targets for infant and neonatal mortality rates (reductions of $61 \%$ and $63 \%$, respectively) were just below the millennium development goal 4 target (67\%). Under five-mortality rates were significantly higher in rural than in urban areas. Children born and raised in rural areas had at least a 38\% higher risk of dying prior to age five compared with their urban counterparts $[2,3]$.

Reducing deaths of newborn and children under 5 years of age became an agenda, worldwide. One of the agenda is sustainable development goals, which set plan for 2030 to end preventable deaths of newborn and children under 5 years of age, with all countries aiming to reduce neonatal mortality to at least as low as 12 per 1,000 live births and under-5 mortality to at least as low as 25 per 1,000 live births [4].

Child mortality can be caused by different factors. Various studies done across regions and worldwide showed different predictors of child mortality. Study done in Nigeria showed, children whose mothers were illiterate and had less than two years of the birth interval had the highest under-5 mortality. Mothers less than 20 years had more chance to lose their children. The child mortality was significantly high in poor households with low birth interval [5].

Study conducted in Nepal identified that family size, birth type, breast feeding status, source of drinking water, and income of mother and marital status as statistically significant with child mortality [6]. Effect of mothers' education, wealth, and exposure to media found significant determinants of child mortality in Pakistan [7].
Birth interval is the practice of waiting between pregnancies. A woman's body needs to rest following pregnancy. After having a baby, it is a good idea to wait at least 18 months before getting pregnant again to maintain the best health for her body and her children. The 18-month rest period is called "birth spacing." When the time between pregnancies is less than 18 months, her body may not be prepared to have a healthy baby. When a woman does not wait at least 18 months between pregnancies, there is an increased risk of having a poor birth outcome such as a premature birth or a low birth weight baby. These conditions are associated with infant mortality and other short-term and long-term health complications [8].

Different studies across globe identified significant association of birth interval and child mortality. Study done in Bangladesh, Nairobi and Afghanistan found that shorter intervals are associated with higher child mortality [9-12]. Demographic and health research identified that the relationship between the preceding birth interval and under-five mortality is highly significant [13]. Systematic review done on low and middle income countries displayed that longer preceding birth intervals had lower odds of mortality than lesser preceding birth interval [14]. In Nigeria, Zimbabwe, rural South Africa and Nepal studies a short preceding interval had the highest risk of child mortality [5-6,15-16]. In Ethiopia, the risk of under one mortality was increased with preceding birth interval less than 24 months. Children born after a proceeding birth interval of 2-3 years and 3 years and above were less likely to have died before their fifth birthday than those born within two years [17-18].

Studies conducted so far in Ethiopia on effect of birth interval on child mortality is limited. Availing updated information on effect of preceding birth interval on child mortality is important to implement effective intervention that focused on child survival. Therefore, the current study identified the effect of preceding birth interval on child mortality in Ethiopia.

\section{Methods}

\section{Setting}

Ethiopia is located in the North Eastern part of Africa, also known as the Horn of Africa. It borders six countries - Eritrea, Djibouti, Somalia, Kenya, South Sudan, and the Sudan. The country occupies an area of 1.1 million square kilometers ranging from $4,620 \mathrm{~m}$ above sea level at RasDashen Mountain to $148 \mathrm{~m}$ below sea level at the Danakil (Dallol) Depression. More than half of the country lies above 1,500 meters. The predominant climate type is tropical monsoon, with temperate climate on the plateau and hot in the lowlands. There are topographic-induced climatic 


\section{Epidemiology international journal}

variations broadly categorized into three: the "Kolla", or hot lowlands up to approximately 1,500 meters above sea level, the "Wayna Degas" or mid lands which range 1,500-2,400 meters above sea level and the "Dega" or cool temperate highlands 2,400 meters above sea level [19].

Projections from the 2007 population and housing census estimate the total population for the year 2015 to be 90 million [15]. Ethiopia is the home of a variety of nations, nationalities, and peoples with more than 80 different spoken languages. The average size of a $\mathrm{HH}$ (household) is 4.7. The pyramidal age structure of the population has remained predominately young with $44.9 \%$ under the age of 15 years, and over half $(52 \%)$ of the population in the age group of 15 and 65 years. The population in the age group of over 65 years accounts for only $3 \%$ of the total population. While the sex ratio between males and females is almost equal, women of reproductive age constitute $23.4 \%$ of the population [2].

Study Design: Cross sectional study.

Study Participants: Non-first births in the 5 years before the 2016 EDHS.

\section{Sampling}

In 2016 EDHS, 10641 births of under five year children were registered. From registered birth, nonfirst births accounted 8460 children. The sample size used in this study was 8460 non-first births in the 5 years before the survey.

The 2016 EDHS sample was stratified and selected in two stages. Each region was stratified into urban and rural areas, which yielded 21 sampling strata. Samples of EAs (enumeration areas) were selected independently in each stratum in two stages. In the first stage, 645 EAs were selected with probability proportional to the EA size and with independent selection in each sampling stratum with the sample allocation. The EA size is the number of residential $\mathrm{HH}$ in the EA. A HH listing operation was implemented in the selected EAs, and the resulting lists of $\mathrm{HH}$ served as the sampling frame for the selection of $\mathrm{HH}$ in the second stage. Some of the selected EAs were large. To minimize the duty of $\mathrm{HH}$ listing, the selected large EAs with more than $200 \mathrm{HH}$ were segmented. Only one segment was selected for the survey with probability proportional to the segment size. HH listing was conducted only in the selected segment. Thus, a 2016 EDHS cluster is either an EA or a segment of an EA.

In the second stage of selection, a fixed number of 28 $\mathrm{HH}$ per cluster were designated with an equal probability systematic selection from the newly created $\mathrm{HH}$ list. The survey interviewer interviewed only the pre-selected HH. No replacements or changes of the pre-selected $\mathrm{HH}$ were allowed in the implementing stages to prevent bias. All women aged 15-49 who are usual members of the selected $\mathrm{HH}$ or who spent the night before the survey in the selected $\mathrm{HH}$ were eligible for the female survey [2].

\section{Data Collection and Measurements}

This study used secondary data of EDHS, 2016. Data were downloaded from DHS (demographic health survey) data set.

Child mortality was the dependent variable of the study. It was measured by two options: yes and no. Child mortality includes data of under-five year children.

Preceding birth interval was used as independent variable of the study. It is number of months since the preceding birth. It was categorized into groups. The categories include: less than 18 months, 18 to 23 months, 24 to 29 months, 30 to 35 months, 36 to 47 months, 48 to 59 months, 60 months and above.

Maternal age, education, wealth index, sex of child, wantedness of the birth, place of residence were used as control variables. These variables were selected by referring different literatures [5-7,12].

Educational status of women: the highest education women attained. It was categorized in to four groups: No education, Primary, Secondary, and Higher.

Wealth Index of HH: This variable in the data set was recorded into five groups: poorest, poorer, middle, richer, and richest.

Place of Residence: was assessed by two options: rural and urban.

Wantedness of Birth: was measured by wanted then, wanted later, and wanted no more.

Sex of child: was measured by two options; male and female

Age at first birth: age was measured in completed year. Age at first birth was gotten by asking at which age childbearing commences.

\section{Data Analysis}

The data analysis was done by using SPSS version 20 . Data were presented by tables, figure and in narration form. Median was calculated for continuous variables. Missing values was handled by using multiple imputation method. Bivariate logistic regression analysis was done assess the effect on preceding birth interval on child mortality. Odds ratio at $95 \%$ CI was computed to assess the strength of association between 


\section{Epidemiology international journal}

dependent and independent variables. Multivariate logistic regression analysis was performed to assess the effect on preceding birth interval on child mortality with adjustments of confounders. Variables with Pvalue $<0.05$ in multivariate analysis was used to declare significance of association. Multicollinearity was checked during multivariate logistic regression analysis. Standard error of significantly associated variables was less than two, showing that there was no multicollinearity.

\section{Results}

\section{Preceding Birth Interval}

Preceding birth interval was calculated for non-first births of under five year children. The median preceding birth interval was 33 months, with minimum and maximum of 7 and 213 months. Highest number of children was found in interval range of 36 to 47 months. Lowest number of children was found in interval range of 48 to 59 months (Figure1).

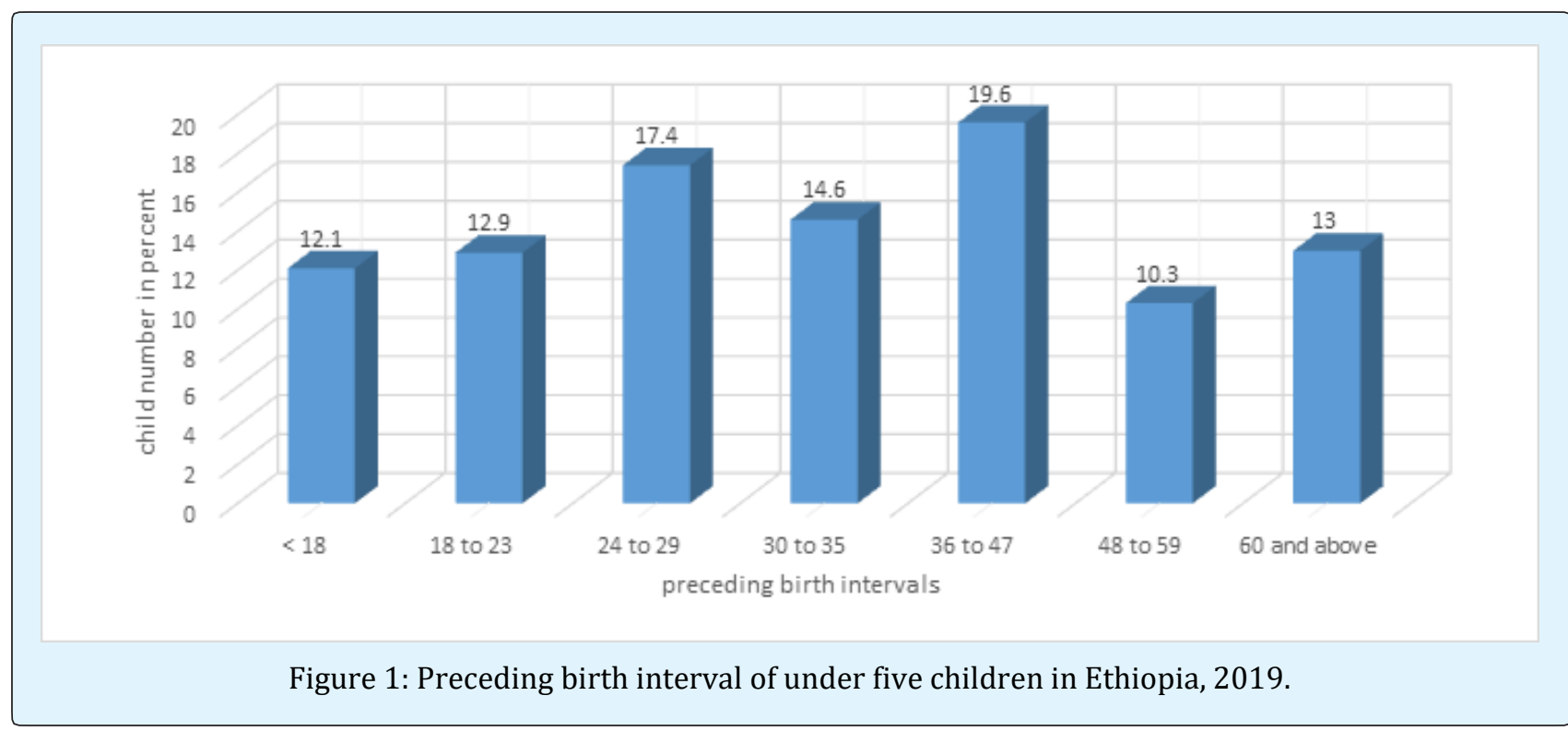

\section{Childhood Mortality by Preceding Birth Interval and Background Variables}

From 8460 non-first births, 495(5.8\%) children were died. In preceding birth interval less than 18 months, $126(1.5 \%)$ children were died. In preceding birth interval of 18 to 23 months, 78(0.8\%), 24 to 29 months, $78(0.8 \%)$ and 30 to 35 months, 64(0.8\%) children were died. In preceding birth interval of 36 to 47 months, 53(0.6\%) children were died. In birth interval of 48 to 59 months, 28(0.3\%) and in 60 months and above, 51(0.6\%) children were died.

Child mortality was higher, 543(6.4\%) in wanted child then group. Male child had higher mortality, $376(6.9 \%)$. In rural setting, 568(6\%) child mortality was occurred. Child morality in no education group accounted, 451(6.6\%). About 287(7.2\%) child mortality was occurred in poorest wealth index category (Table 1).

\begin{tabular}{|c|c|c|}
\hline Variables & \multicolumn{2}{|c|}{ Childhood mortality } \\
\hline Wanted last child & Yes (\%) & No (\%) \\
\hline Wanted then & $543(6.4)$ & $7946(93.6)$ \\
\hline Wanted later & $54(3.6)$ & $1437(96.4)$ \\
\hline Wanted no more & $38(5.7)$ & $632(94.3)$ \\
\hline Sex of child & & $5107(93.1)$ \\
\hline Male & $376(6.9)$ & $4899(94)$ \\
\hline Female & $259(6)$ & $1907(96.6)$ \\
\hline Type of place of residence & & $8099(94)$ \\
\hline Urban & $67(3.4)$ & \\
\hline Rural & $568(6)$ & $6387(93.4)$ \\
\hline
\end{tabular}




\section{Epidemiology international journal}

\begin{tabular}{|c|c|c|}
\hline Primary & $140(5.2)$ & $2538(94.8)$ \\
\hline Secondary & $37(5)$ & $697(95)$ \\
\hline Higher & $7(2.8)$ & $384(98.2)$ \\
\hline Wealth index combined & & \\
\hline Poorest & $287(7.2)$ & $3706(92.8)$ \\
\hline Poorer & $112(6.3)$ & $1670(93.7)$ \\
\hline Middle & $80(5.5)$ & $1386(94.5)$ \\
\hline Richer & $81(6.8)$ & $1227(93.8)$ \\
\hline Richest & $75(3.6)$ & $2017(96.4)$ \\
\hline
\end{tabular}

Table 1: Child mortality by background variables in Ethiopia, 2019.

\section{Association between Child Mortality and Preceding Birth Interval}

Child whose preceding birth interval less than 18 months was about three times more likely to die than child with birth interval of 60 months and above(AOR= 2.769; CI $95 \%(1.956,3.921))$. Child whose preceding birth interval was between 18 and 23 months was 1.5 times more likely to die than child with birth interval of 60 months and above (AOR=1.500; $95 \%$ CI (1.038, 2.168)). Child whose preceding birth interval was between 36 and 47 months was $33.7 \%$ less likely to die than child with birth interval of 60 months and above (AOR=0.663, $95 \%$ CI $(.446, .986)$ (Table 2$)$.

\begin{tabular}{|c|c|c|c|c|c|c|}
\hline \multirow{2}{*}{ variable } & \multicolumn{3}{|c|}{ Child mortality } & \multirow{2}{*}{ COR(95 \% CI) } & \multirow{2}{*}{ AOR(95 \% CI) } & \multirow{2}{*}{ p-value } \\
\hline & & Yes & No & & & \\
\hline \multirow{7}{*}{$\begin{array}{l}\text { Preceding birth } \\
\text { interval in months }\end{array}$} & Less than 18 & $126(12.3 \%)$ & $901(87.7 \%)$ & $2.946(2.103,4.127)$ & $2.769(1.956,3.921)$ & 0.000 \\
\hline & 18 to 23 & $78(7.1 \%)$ & 1013(92.9\%) & $1.606(1.123, .298)$ & $1.500(1.038,2.168)$ & 0.031 \\
\hline & 24 to 29 & $94(6.4 \%)$ & $1377(93.6 \%)$ & $1.463(1.049,2.042)$ & $1.354(0.961,1.907)$ & 0.083 \\
\hline & 30 to 35 & $64(5.2 \%)$ & $1173(94.8 \%)$ & $1.136(.783,1.649)$ & $1.066(0.729,1.559)$ & 0.742 \\
\hline & 36 to 47 & $53(3.2 \%)$ & $1607(96.8 \%)$ & $0.711(.481,1.051)$ & $0.663(0.446,0.986)$ & 0.043 \\
\hline & 48 to 59 & $28(3.2 \%)$ & $844(96.8 \%)$ & $0.683(.431,1.080)$ & $0.648(0.408,1.030)$ & 0.066 \\
\hline & 60 and above & $51(4.6 \%)$ & 1051(95.4\%) & 1 & 1 & \\
\hline
\end{tabular}

Table 2: Association between child mortality and preceding birth interval in Ethiopia, 2019

Adjusted with the maternal age at birth, educational status of women, wealth index of HH, sex of the child, place of residence, child wanted.

\section{Discussion}

This study tried to identify the effects of preceding birth interval on child mortality. Based on the finding of this study, preceding interval was associated with child mortality. Child whose preceding birth interval less than 18 months was more likely to die than child with birth interval of 60 months and above. In addition, Child whose preceding birth interval was between 18 and 23 months was more likely to die than child with birth interval of 60 months and more. This is in line with the following study, which found that shorter intervals are associated with higher mortality [10]. Study conducted in Afghanistan identified that a preceding birth interval less than 18 months was associated with a two-fold increase in mortality risks compared with lengthened intervals of 36 months or longer [11]. The demographic health survey study done in developing countries showed highly significant association between the preceding birth interval and under-five mortality [13]. In Bangladesh, very short birth intervals of less than 21 months were associated with increased neonatal mortality [12]. In Nigeria and Zimbabwe, under-5 deaths were more in less than two years of birth interval $[14,15]$. Study in Ethiopia displayed that the risk of under one mortality was increased with preceding birth interval less than 24 months [18]. other studies done in Ethiopia also revealed that children born after a preceding birth interval of 2-3 years and 3 years and above were significantly less likely to have died before their fifth birthday than those born within two years[17]. United nation children's fund also displayed that short birth interval children have a 50 percent greater risk of dying between the ages of one and four years than do children born after a longer birth interval [20].

The mechanism linking birth interval length and child mortality are maternal depletion, sibling competition and transmission of infectious diseases [21]. The maternal depletion hypothesis argues that short birth intervals do not allow women to fully recover their nutritional stores after a prior birth, which may lead to diminished fetal growth as their bodies compete with that of the fetus for nourishment. The sibling competition hypothesis claims that closely 


\section{Epidemiology international journal}

spaced siblings will compete for similar resources from their parents. The competition may lead to weaker immune system index children, placing them at greater risk of infection and even death. The disease transmission hypothesis argues that closely spaced sibling will be more likely to pass infections to one another and because a younger child will generally have less developed immune system than its older siblings, he or she will be more likely to die at younger [21].

The reason for the association between short preceding birth interval and child mortality could be when birth interval is shorter, there is an increased risk of having a poor birth outcome such as a premature birth or a low birth weight baby. These conditions can threaten the health of the child and the mother. This finding calls that birth spacing should be an agenda of the community and concerned bodies.

Child whose preceding birth interval was between 36 and 47 months was less likely to die than child with birth interval of 60 months and above. This is comparable with study done in Afghanistan, which indicated that children with a previous birth interval of at least 60 months have a higher risk of dying from certain causes of diseases than children with a previous birth interval of 24-35 month [11]. The mechanism linking longer birth interval length and child mortality needs further investigation. Further studies should be done by incorporating the effects of longer birth interval on child mortality.

\section{Limitation of the Study}

As many questions were dealt with past life experience, recall bias can happen. The mothers may remember major life events; though, they might not recall many variables such as age during child birth and exact birth interval. Confounding variables included in current study are based on their effect as evidenced by most published researches but other variable that could have an effect are not included. Thus, interpretation of the study should consider these limitations.

\section{Conclusion}

The preceding birth interval and child mortality was significantly associated. Child whose preceding birth interval less than 18 months was more likely to die than child with birth interval of 60 months and above. Child whose preceding birth interval was between 18 and 23 months was more likely to die than child with birth interval of 60 months and more. Child whose preceding birth interval was between 36 and 47 months was less likely than to die than child with birth interval of 60 months. Health education on effect of short preceding birth interval should be given by health workers.
Promotion of family planning should be strengthened at all levels.

\section{Declaration}

\section{Ethical approval and consent to participate}

This complies with national and international guideline [2]. In DHS, verbal informed consent is taken for women questionnaire. Verbal informed consent is sought by the interviewer reading a prescribed statement to the respondent and recording in the questionnaire whether or not the respondent consented. Then, the interviewer sign his/her name attesting to the fact that he/she read the consent statement to the respondent. Thus, the respondent is not asked to sign their name as the interviewer has attested that the procedure was followed. The purpose of current analysis was sent to DHS organization. Permission to download and to use data was obtained from the DHS organization [22].

Availability of data and materials: The data that support the findings of this study are available from DHS but restrictions apply to the availability of these data, which were used under license for the current study, and so are not publicly available.

Authors' contribution: TL designed the study protocol, analyzed the data and drafted the paper. TL read and approved the final manuscript.

\section{References}

1. (2019) Under-five mortality.

2. Ethiopia Demographic and Health Survey (2016) Addis Ababa, Ethiopia, and Rockville, Maryland, USA: CSA and ICF: Central Statistical Agency (CSA) [Ethiopia] and ICF.

3. Central Statistical Authority (2011) Ethiopian demographic and health survey 2011. Addis Ababa, Ethiopia: CSA.

4. (2015) The 2030 agenda for Sustainable development goals. New work.

5. Biradara R, Patelb KK, Prasadb JB (2018) Effect of birth interval and wealth on under-5 child mortality in Nigeria. Clin Epidem Global Heal 5: 5.

6. Pathak A (2017) socioeconomic determinants of child mortality: analysis of the 2011 Nepal demographic and health survey. Finland: University of Eastern Finland.

7. Rabbani S, Qayyum A (2015) Comparative Analysis of Factor Affecting Child Mortality in Pakistan: 32. 


\section{Epidemiology international journal}

8. Family planning program. Birth spacing. Delaware Health and Social Services Division of Public Health. 2011.

9. Davanzo J, Hale L, Razzaque A, Rahman M (2008) The effects of pregnancy spacing on infant and child mortality in Matlab, Bangladesh: How they vary by the type of pregnancy outcome that began the interval. Popul Stud 62(2): 131-154.

10. Fotso Jc, Cleland J, Mberu B, Mutua M, Elungata P (2013) Birth Spacing And Child Mortality: An Analysis of Prospective Data From The Nairobi Urban Health And Demographic Surveillance System. J Biosoc Sci 45(6): 779-798.

11. Rasooly MH, Saeed KMI, Noormal B, Aman I, Arnold F, et al. (2013) The Effect of Birth Intervals on Causes of Under-Five Mortality in Afghanistan. Maryland, USA: ICF International Calverton, p: 25.

12. Jonge HCd, Azad K, Seward N, Kuddus A, Shaha S, et al. (2014) Determinants and consequences of short birth interval in rural Bangladesh: a cross-sectional study. BMC Pregnancy and Childbirth 14(427).

13. Rutstein SO (2008) Further Evidence of the Effects of Preceding Birth Intervals on Neonatal, Infant, and Under-Five-Years Mortality and Nutritional Status in Developing Countries: Evidence from the Demographic and Health Surveys. demographic and health research. USA, 99: 86.

14. Kozuki N, Walker N (2013) Exploring the association between short/long preceding birth intervals and child mortality: using reference birth interval children of the same mother as comparison. BMC Public Health 13(3).
15. Kembo J, Ginneken JKV (2009) Determinants of infant and child mortality in Zimbabwe: Results of multivariate hazard analysis. Germany: Max Planck Institute for Demographic Research Konrad-Zuse Str: 20.

16. Houle B, Stein A, Kathleen K, Madhavan S, Collinso $M$, et al. (2013) Household context and child mortality in rural South Africa: the effects of birth spacing, shared mortality, household composition and socio-economic status. Int J Epidem 42(5): 1444-1454

17. Gebretsadik S, Gabreyohannes E (2016) Determinants of Under-Five Mortality in High Mortality Regions of Ethiopia: An Analysis of the 2011 Ethiopia Demographic and Health Survey Data. Int J Popul Res: 7.

18. Dadi AF (2014) Short birth intervals less than 2 years double under-one mortality in Ethiopia: Evidence from a meta- analysis. Sci J Public Health 2(6): 589-595.

19. Child spacing.

20. Conde-Agudelo A, Rosas-Bermudez A, Castaño F, Norton MH (2012) Effects of Birth Spacing on Maternal, Perinatal,Infant, and Child Health: A Systematic Review of Causal Mechanisms. Stud Fam Plann 43(2): 93-114.

21. Gibbs MC, Wendt A, Peters S, Hogue CJ (2012) The impact of early age at first childbirth on maternal and infant health. Paediatric and Perinatal Epidemiol 26(1): 259-284.

22. DHS program (2019) Protecting the privacy of DHS survey respondent. 